\title{
Suitable Interpretation of Skin Prick Test and Biomedical Guidance Leads to a Better Clinical State in Atopic Individuals with High Indoor Permanence: Possible Therapeutic Implications
}

\author{
Bernardo Yassunobu Nakamatsu ${ }^{1,+}$, Archangelo Padreca Fernandes $1,+$, Débora da Silva 1, ${ }^{\dagger}$, \\ Ludimila Souza Santos ${ }^{2}$, Thamires Rodrigues de Sousa ${ }^{2}$ and Jefferson Russo Victor 1,2,3,*(D) \\ 1 Faculdades Metropolitanas Unidas (FMU), Sao Paulo 04505-002, Brazil; b.nakamatsu@uol.com.br (B.Y.N.); \\ archangelo.fmu@gmail.com (A.P.F.); desilva@portoseguro.org.br (D.d.S.) \\ 2 Laboratory of Medical Investigation LIM 56, Division of Dermatology, School of Medicine, University of Sao \\ Paulo (FM-USP), Sao Paulo 05403-000, Brazil; ludisantos3110@gmail.com (L.S.S.); \\ sousarthamires@gmail.com (T.R.d.S.) \\ 3 School of Medicine, Universidade Santo Amaro (UNISA), Sao Paulo 04829-300, Brazil \\ * Correspondence: victorjr@usp.br; Tel.: +55-11-30-617-499; Fax: +55-11-30-817-190 \\ + These authors contribute equally.
}

Citation: Nakamatsu, B.Y; Fernandes, A.P.; da Silva, D.; Santos, L.S.; de Sousa, T.R.; Victor, J.R. Suitable Interpretation of Skin Prick Test and Biomedical Guidance Leads to a Better Clinical State in Atopic Individuals with High Indoor Permanence: Possible Therapeutic Implications. Biologics 2021, 1 , 222-230. https://doi.org/10.3390/ biologics1020014

Academic Editor: Francois Niyonsaba

Received: 20 July 2021

Accepted: 31 August 2021

Published: 3 September 2021

Publisher's Note: MDPI stays neutral with regard to jurisdictional claims in published maps and institutional affiliations.

Copyright: (c) 2021 by the authors. Licensee MDPI, Basel, Switzerland. This article is an open access article distributed under the terms and conditions of the Creative Commons Attribution (CC BY) license (https:// creativecommons.org/licenses/by/ $4.0 /)$.

\begin{abstract}
Indoor conditions contribute to allergen sensitization and multiple allergens reactivity, mainly for inhaled allergens. This study analyzes if Skin Prick Test (SPT) combined with efficient individual biomedical guidance about allergy development's social, biological, and environmental aspects can yield a better clinical state with therapeutic implications for atopic individuals with high indoor permanence. We recruited atopic and non-atopic volunteers (clinically and in vitro diagnosed) with indoor permanence above $15 \mathrm{~h}$ per day and without previous SPT evaluation. The SPT and serum anti-allergen IgE analyses were performed individually in person, demonstrated, and discussed by the practitioners. Six months after, SPT and specific IgE titers determination were repeated, and a questionnaire to evaluate the effectiveness of the practitioner's orientation was performed. After six months, $14 \%$ of atopic volunteers reported changes in their social habits, $30 \%$ said that they avoid the development of allergies clinical symptoms, and $68 \%$ reported a substantial improvement in their health after being informed mentored about their allergen reactivity. The control non-atopic group, as expected, reported no changes in social habits, the maintenance of total avoidance of allergic symptoms, and almost no improvement of their health. Reduced SPT and serum allergen-specific IgE titers were detected in the atopic individuals corroborating with questionnaire results. Our results indicated that SPT, followed by an individual and efficient discussion about the main biomedical aspects of allergy development, could exert a pronounced therapeutic role in allergy development by high indoor permanence individuals.
\end{abstract}

Keywords: skin prick test; allergy; indoor; human; therapeutic

\section{Introduction}

Large urban centers have influenced citizens' customs, which has remained more time in indoor conditions. This feature directly affects the pattern of allergens to which these individuals are being exposed and favors the development of clinical allergy symptoms. Because of its growing importance in public health, all strategies that can improve the control of human allergy development must be considered.

Many medical devices are available to diagnose, prevent, treat, and monitor allergies, yielding sophisticated diagnostic and management processes that require integrated health care systems [1] that are not frequent in developing countries. 
In this context, currently, testing for allergen-specific $\operatorname{IgE}$ is helpful, indicating the presence or absence of IgE sensitization in suspected allergic conditions, but other in vitro and in vivo tests were frequently used [2]. The skin prick test (SPT) represents the first level of diagnosing IgE-mediated allergy, and it is safe, has high sensitivity, reasonable specificity, and can exert an educational character [3].

When performing an SPT, an allergen is introduced through a lancet into the skin where mast cells degranulate due to the cross-linking of anti-allergen IgE bound to their membrane receptors. This degranulation leads to the immediate release of histamine and other mediators, inducing a cutaneous response, clinically characterized by a wheal that can be measured, and its size is directly related to the degree of cutaneous sensitivity. This test has particular importance in diagnosing allergies mediated by inhaled allergens $[4,5]$.

To avoid clinical symptoms of allergy reactions is necessary to identify allergens that can induce clinical symptoms on each individual [6]. We consider as crucial that the atopic individual might know specifically the allergens that can trigger its allergic reactions. Furthermore, the growing indoor habit in urban zones can intensify the exposure and development of allergies, difficult to identify specific allergens. We also need to consider that SPT usage in public health systems is very limited in developing countries.

Thus, we hypothesized that the acquisition of indoor habits by atopic individuals who did not have access to an SPT and could not identify the main allergens that can induce clinical manifestations can lead to an allergy development cycle implicating a public health problem.

Therefore, we aimed to perform a study to evaluate if SPT combined with biomedical counseling can exert some therapeutic effect on allergy development, mainly for those atopic individuals with high indoor permanence.

\section{Materials and Methods}

\subsection{Study Population}

Initially, we disclose our study in a randomized manner using social networks during the second half of 2017. Questionnaires to collect sociodemographic information were administered to 1256 volunteers interested in the survey to select those representing a typical urban life profile that we wanted to evaluate. We also considered the place of residence of the volunteers to cover the five central urban regions of the city of Sao Paulo (Sao Paulo state-Brazil).

After analyzing the data obtained with the primary analyses, during 2018 and 2019, we could recruit 157 atopic volunteers that declare to have previous laboratory diagnoses (IgE-specific titers to allergens). These volunteers were evaluated to confirm their clinically allergic state (confirmed by medical consultation where clinical symptoms of allergies, including rhinitis and asthma, could be evidenced) and, those that had the allergic state confirmed were selected with the following inclusion criteria: those who were born and always lived in urban zones of the Sao Paulo city; that still inhabit residences with access to the sewage network; that had never been submitted to skin SPT; and that remains at least $15 \mathrm{~h}$ a day indoors with air conditioning.

As exclusion criteria, we consider volunteers with severe eczema or dermographism or who used any of the following within 15 days before the test: antihistamines, glucocorticosteroids, or other systemic drugs that can influence SPT results. After the application of these inclusion and exclusion requirements, our final number of atopic volunteers considered in this study was 132 with a mean age of 42.53 years $( \pm 3.78)$, from both sexes ( 72 females and 60 males) and with a mean of indoor permanence time of $15.78 \mathrm{~h}( \pm 0.52)$. Due to specific requirements for inclusion and exclusion in the study, the number of participants could be considered adequate for this study. Volunteers were instructed to maintain the same dose and frequency of anti-allergic medications during the assessment period as they used previously.

As a control group, we recruited 152 volunteers that declare to be non-atopic when answering the questionnaire. These individuals were evaluated to confirm their clinically non-atopic state (confirmed by medical consultation where clinical symptoms of allergies 
were absent). Their non-atopic state was also confirmed by the absence of skin prick test (SPT) reactivity to all tested allergens and undetectable anti-allergen IgE titers in serum. Volunteers with the confirmed non-atopic state were submitted to the same inclusion criteria adopted for the atopic group (those who was born and always lived in urban zones of the Sao Paulo city; that still inhabit residences with access to the sewage network; that had never been submitted to skin SPT; and that remains at least $15 \mathrm{~h}$ a day indoors with air conditioning).

After the application of the inclusion requirements, our final number of non-atopic volunteers considered in this study was 102 with a mean age of 33.71 years $( \pm 3.11)$, from both sexes ( 63 females and 39 males) and with a mean of indoor permanence time of $13.45 \mathrm{~h}$ $( \pm 0.47)$.

All numerical parameters obtained after grouping our volunteers were compared between atopic and non-atopic individuals, and no statistical significance was observed.

The local and national ethics committees approved this study (Certificate of Presentation of Ethical Appreciation: 80280417.8.0000.8114), and all volunteers obtained informed consent. All the procedures, including humans, are following the Helsinki Declaration.

\section{2. $S P T$}

SPTs were performed following European standards [7] and using an adapted panel of allergens that included a profile of Brazilian allergens. We tested allergens with origin in 16 different species grouped in 7 different extracts (Supplementary Table S1). Briefly, one drop of each allergen extract, histamine (positive control), or the allergen diluents (negative control) provided by IPI ASAC was applied to the volar forearm. A superficial skin puncture was made through each allergen or control drop using a single-head metal lancet (Alko, Sao Paulo, Brazil) without inducing bleeding. After $15 \mathrm{~min}$, the results were obtained by measuring the transverse diameter of each wheal reaction. Only volunteers with a positive control wheal diameter greater than $5 \mathrm{~mm}$ were considered in this study. Wheals with a diameter of $5 \mathrm{~mm}$ higher than the negative control were deemed to be positive results. All atopic patients (reactive to at least one tested allergen) were conducted to an individual in-person discussion about biomedical aspects and their relation to occupational, social, and environmental aspects related to their skin reactivity by an allergist. This discussion aimed to demonstrate the primary sources of the allergens that induce SPT reactivity, avoid exposition and apply cleaning methods to reduce allergens exposure. This orientation was performed using World Allergy Organization (WAO) professional standards on allergen avoidance (available in: https:/ / www.worldallergy.org/education-and-programs / education/allergic-disease-resource-center/professionals/allergen-avoidance/ accessed on 31 August 2021).

Briefly, volunteers received precise information about humidity control, the use of acaricides, good care of beds and bed covers, washing procedures, use of a high-efficiency vacuum cleaner, pets washing, air filtration, integrated pest management (IPM), how to preventing pest access, encasing pest food supplies and moisture control.

\subsection{Blood Samples Obtainment}

Blood samples were obtained in the initial stage of our study to confirm volunteers' atopic state and generate pieces of evidence about allergen-reactivity to atopic individuals. When performing the therapeutic effectiveness evaluation, an additional blood sample was obtained from each individual. The blood samples were centrifuged, and the serum was fractionated and stored at $-80^{\circ} \mathrm{C}$ until anti-allergen $\mathrm{IgE}$ determination.

\subsection{Serum Anti-Allergen IgE Determination}

According to the manufacturer's instructions, serum-specific IgE antibodies have been measured with a multiplex immunoblot assay (EUROLINE Inhalation 2-EUROIMMUN AG, Lubek, Germany). The tested extracts are described in Supplementary Table S2. Briefly, the strips were incubated with patients' sera, and, after the washing step, the strips were incubated with alkaline phosphatase-conjugated anti-human. After the second washing 
step, the strips were incubated with the chromogen/substrate solution. The reaction was stopped by washing, and the strips were evaluated with the EUROLineScan software (EUROIMMUN, Lubek, Germany) to obtain quantitative results.

The intensity of the bands was measured and converted into a score from zero to six was divided into the following concentrations (all expressed in $\mathrm{kU} / \mathrm{L}$ ): class $0<0.35$; class $1<0.7$; class $2<3.5$; class $3<17.5$; class $4<50.0$; class $5<100.0$; class $6 \geq 100.0$. According to the manufacturer's instructions, individuals who have a reactivity equal to or higher than grade 2 frequently develop clinical symptoms. Therefore, because we aimed to generate additional biological evidence for symptomlessness reporting, we consider only individuals with reactivity equal to or above class 2 in our analyses.

\subsection{SPT Therapeutic Effectiveness Evaluation}

Six months after the volunteers' recruitment to perform SPT, Serum IgE evaluation, and individual in-person discussion about biomedical aspects and their allergic reactivity, all tested volunteers were reconvened to answer questions about the therapeutic effectiveness of the adopted procedure. The applied questions were the following: i-Have you changed your social habits after SPT results?; ii-Could you avoid the development of allergies entirely?; iii-The result of the SPT collaborated with an improvement of your health?

The volunteers were guided to "yes or no" answers, and the parameters used to validate each question are described below:

Question i Yes, it was considered valid only if at least one regular social habit was wholly avoided during the analysis period.

Question ii Yes, it was considered if the volunteer did not develop any of the previously described clinical symptoms of allergies during the analysis period.

Question iii Yes, it was considered if the volunteer could precisely describe a health improvement related to decreased use of continuous medication, decreased workday loss, and the implementation of a previously avoided activity due to allergy manifestations.

All volunteers who answer "yes" in at least one of the performed questions were submitted to a second validation question: Can you confirm that the observed alterations are due to the knowledge gained from SPT? Moreover, only those that answered "yes" to this second question were included in their result group. These questions were previously validated in a local therapeutic evaluation study comparing allergic and non-allergic individuals submitted to SPT. After answering the questions, the volunteers were subjected to a new medical consultation to confirm that the items were answered correctly. The supplemental Figure S1 illustrates the therapeutic effectiveness evaluation scheme.

\subsection{Statistical Analyzes}

The data obtained in the SPT and IgE evaluations were not statistically compared because the non-atopic group presented only undetectable/negative results, which made the difference between the groups evident. A similar profile was observed in the results obtained with the therapeutic effectiveness evaluation questionnaire.

\section{Results}

SPT results demonstrated that all atopic subjects with high indoor permanence are reactive to inhaled allergens extracts and demonstrated that the most frequent reactivity could be detected in response to house dust mite extract (HDM, n: 122, 92.4\%), followed by fungi (n: $80,60.6 \%$ ), grass (n: 60, 45.4\%), cat (n: 57, 43.1\%), dog (n: 56, 42.4\%), cockroaches (n: 29, 21.9\%), chicken feather (n: 15, 11.3\%-Figure 1A). Evaluating the simultaneous reactivity of our atopic subjects, we could observe that all of them (n: 132, 100\%) co-reacts to at least two allergens extracts and most from three to six different allergens extracts (Figure 1B). Some of them could simultaneously react to all tested extracts. As the absence of SPT reactivity was an inclusion criterion in the non-atopic group, the results obtained were all negative and not shown. 


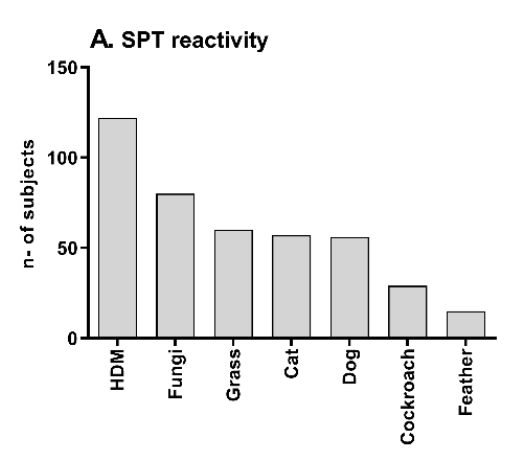

C. Specific IgE reactivity

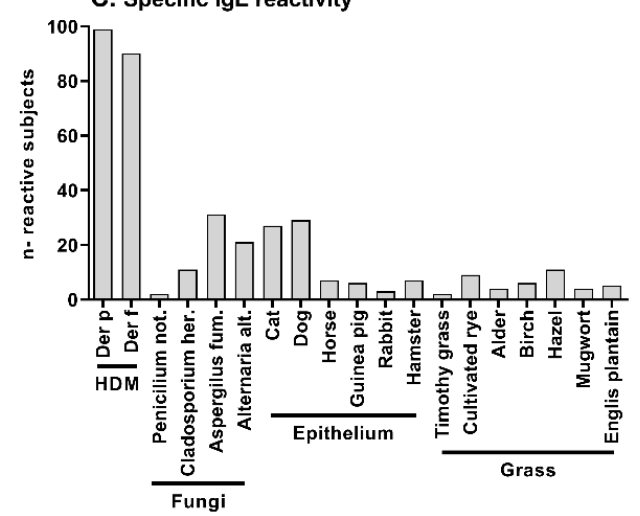

B. SPT co-reactivity

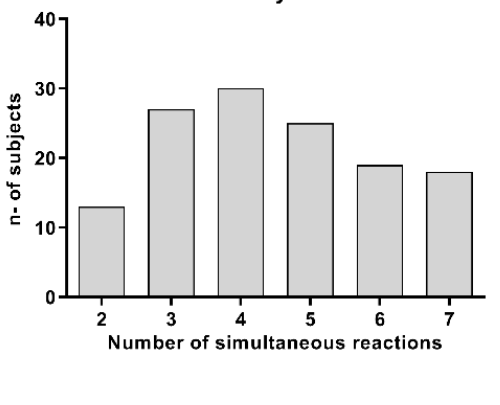

D. Specific IgE reactivity

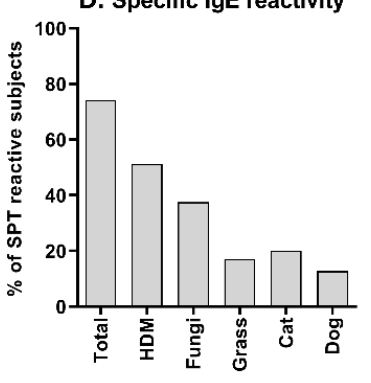

\section{E. Prophylactic effectiveness of SPT}
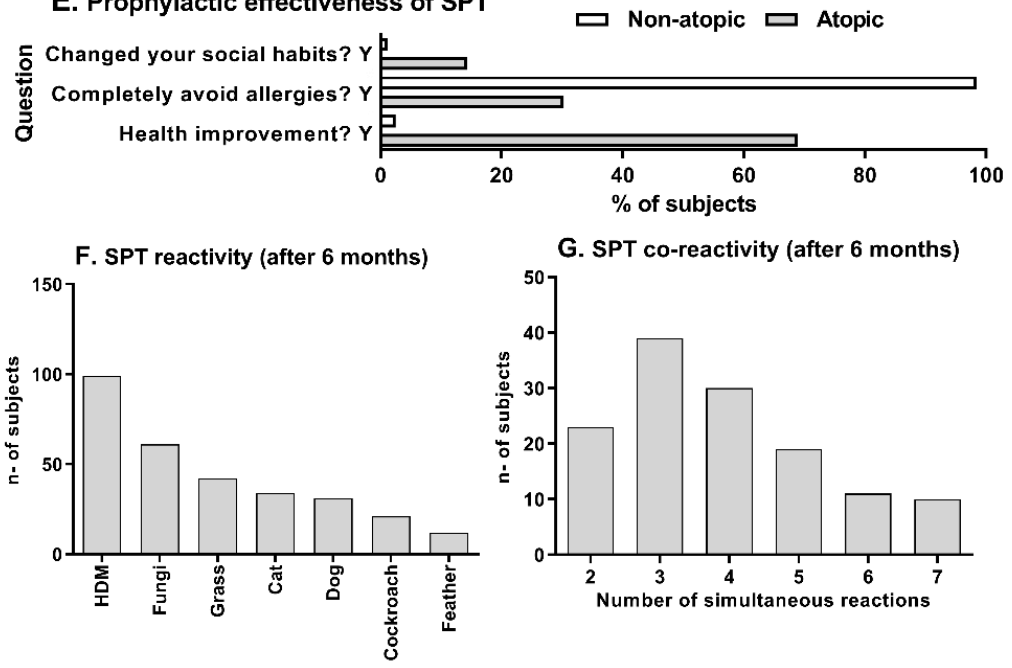

G. SPT co-reactivity (after 6 months)
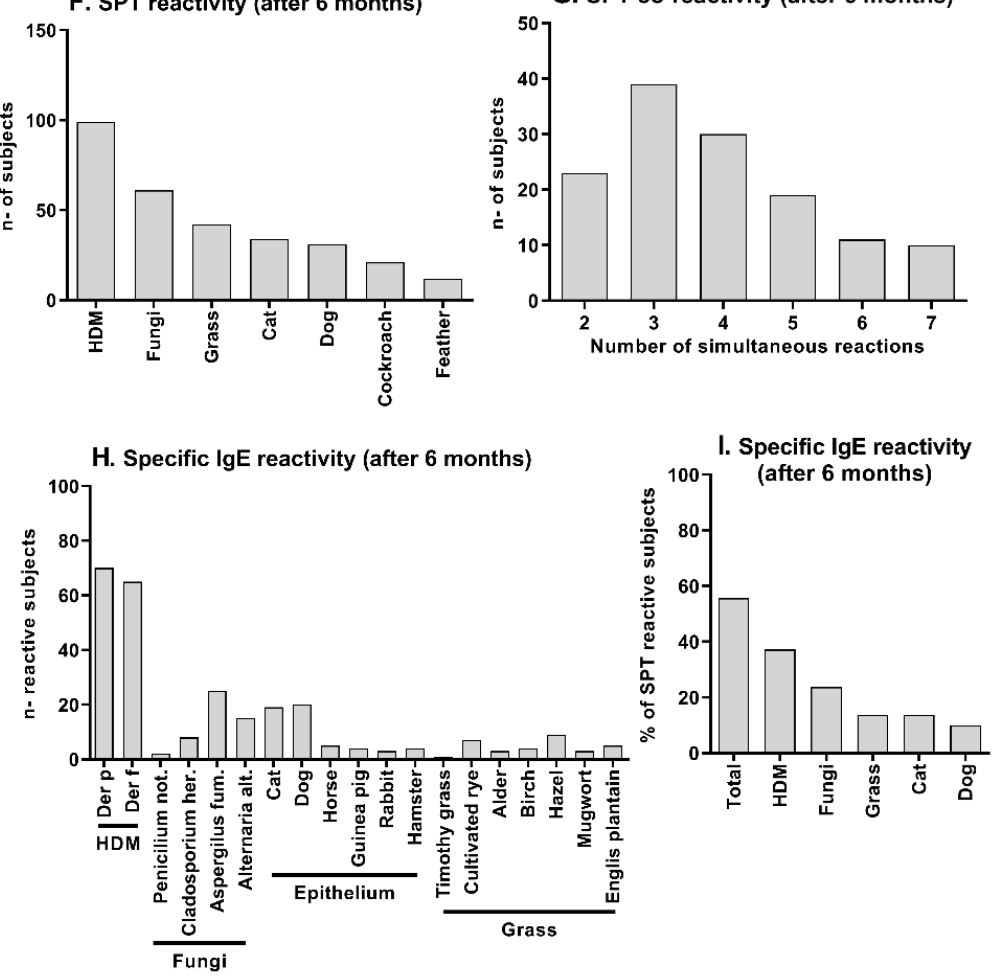

Figure 1. SPT reactivity, serum specific-IgE titers, and individual orientation effectiveness. Atopic (n: 132) or non-atopic (n: 102) volunteers with high indoor permanence were submitted to SPT, and the 
number of reactive and co-reactive atopic subjects was evaluated before personal social/biological/environmental orientation (A-D) and six months later $(\mathbf{F}-\mathbf{I})$. SPT reactivity $(\mathbf{A}, \mathbf{F})$ was evaluated in pooled extracts with the following composition, HDM: Dermatophagoides farinae and Dermatophagoides Pteronyssimus, Fungi: Aspergillus fulmigatus, Penicillium notatum, Alternaria Alternata, and Cladosporium herbarum, Grass: Alder, Birch, Hazel and Cultivated rye, Cat: Felis domesticus, Dog: Canis Familiaris, Cockroach: Blatella germanica and Periplaneta americana, Feather: Chicken and Canary bird. The number of simultaneous reactions for SPT is also demonstrated (B,G). Specific-IgE serum levels were assessed by immunoblot, and the number of atopic subjects that reach a clinical symptoms-related IgE titer was demonstrated to each tested allergen $(\mathbf{C}, \mathbf{H})$ or grouped similar to SPT extracts arrangement $(\mathbf{D}, \mathbf{I})$. The absence of reactivity in these parameters was an inclusion criterion recruiting non-atopic subjects, which was maintained until the second evaluation (six months later). These results were not illustrated. The middle panel (E) shows the frequency of validated answers "yes" to the three main questions performed at the therapeutic effectiveness evaluation interview for atopic and non-atopic subjects is shown in the middle panel (E).

Blood samples of all atopic volunteers were also analyzed to evaluate the reactivity profile of specific IgE antibodies against the most frequent allergens detected in the SPT. In Figure 1C, we can observe the number of individuals who reach allergen-specific IgE titers related to clinical symptoms to each of the tested allergens. The IgE reactivity to HDM, Fungi, Epithelium, and Grass was most frequent (Figure 1C). Grouping these IgE reactivities as SPT evaluation and evaluating its frequency, we could observe that $74.2 \%$ of the individuals reach clinical symptoms-related IgE titers and the rate of HDM reactivity was 51.2\%, Fungi 37.5\%, Grass 17.1\%, Cat 20.1\%, and Dog 12.8\% (Figure 1D) suggesting a similar profile to those observed on SPT. As the absence of anti-allergen IgE reactivity was an inclusion criterion in the non-atopic group, the results obtained were all negative and not shown in the figure.

Six months later, we performed the questionnaire and analyze volunteers' answers about allergy development. We could observe that 19 atopic (14.3\%) and 1 non-atopic $(1.02 \%)$ subject reported changes in their social habits, 40 atopic $(30.3 \%)$ and 95 nonatopic $(96.9 \%)$ subjects reported avoidance of clinical symptoms completely, and 91 atopic $(68.9 \%)$ and 3 non-atopic $(3.06 \%)$ reported a substantial improvement on their health after been informed about their reactivity to allergens (Figure 1E). In this aspect, we evaluate the results obtained from non-atopic volunteers to control the questionnaire efficacy collecting trustworthy answers and to eliminate social habits bias that could generally influence volunteers.

After collecting questionnaire answers, we repeated the SPT and analyzed the reactivity profile of specific IgE antibodies against the same allergens evaluated before the questionnaire on both groups. At this time, SPT results demonstrated that the atopic subjects maintained a similar profile of reactivity to allergens extracts with a reduction in the frequencies, with the respective reactivities: house dust mite extract (HDM, n-99, $75.5 \%$ ), fungi (n: $61,46.2 \%$ ), grass (n: $42,31.8 \%$ ), cat (n: 34, 25.7\%), dog (n: 31, 23.4\%), cockroaches (n: 21, 15.9\%), chicken feather (n: 12, 9.0\%-Figure 1F). A comparative table between the results obtained at the initial evaluation and six months later can be observed on supplementary material (Table S3). We could also observe that the simultaneous reactivity to at least two extracts observed on atopic subjects was maintained (n: 132, 100\%). An augmented frequency of atopic individuals that co-react to two and three allergens and a diminished frequency of atopic individuals that co-reacts to five, six, and seven allergens were observed (Figure 1G) compared to the initial observations. The absence of SPT reactivity was maintained in all non-atopic individuals, and this data was not shown.

In Figure 1F, we can also observe that the number of atopic individuals that reach allergen-specific IgE titers is also reduced compared to the initial observation, but the reactivity profile was maintained with HDM, Fungi, Epithelium, and Grass as the most frequent (Figure 1F). Performing the same grouping of these results as demonstrated in 
the initial results, we could observe that the frequency of all evaluated allergens was reduced, with $55.6 \%$ of the atopic individuals reaching clinical symptoms-related IgE titers (Figure 1G). Between those atopic individuals, the rate of HDM reactivity was 37.2\%, Fungi $23.7 \%$, Grass $13.7 \%$, Cat $13.7 \%$, and Dog 9.8\% (Figure 1G). The absence of anti-allergen IgE reactivity was maintained in all non-atopic individuals, and this data was not shown.

\section{Discussion}

Several studies evaluating SPT reactivity can be found in the literature, but they usually consider geographic [8-10], unhygienic living conditions [11], or smoking [12] and not specific urban behaviors as we evaluated here.

In a well-designed study that evaluated the profile of SPT reactivity to environmental aeroallergens in 35 centers in 15 developed European countries, the most frequent sensitizing allergen was HDM, with a prevalence ranging from $50 \%$ to $70 \%$ in atopic individuals [9], a lower frequency compared to our results.

This observation suggests that the pattern of SPT reactivity detected in our results is slightly higher than the literature, suggesting that the high indoor permanence can be related to polyallergic responsiveness.

Some studies had also evaluated the capacity of individuals to react to more than one allergen, the co-reactivity or poly-reactivity simultaneously. In 2008, the SPT co-reactivity of Brazilian individuals was assessed, and this work demonstrates that nearly $50 \%$ of the evaluated individuals develop a co-reactivity to more than one allergen [8]. A similar approach was made in Pakistan, where the researchers evidenced a co-reactivity of nearly $78 \%$ [13]. Our results demonstrated a co-reactivity of $100 \%$ of the atopic individuals allowing our volunteers to be characterized as polyallergic individuals.

In an extensive revision of the literature (from 1970 to 2005), it was observed that in vivo evidence of cutaneous reactivity by SPT cannot accurately predict the capacity of an individual to develop clinical allergy manifestations. Still, a good relationship between its results and allergy development is a consensus in the literature [3].

Regarding the SPT therapeutic effectiveness on allergy development, the select atopic individuals must never perform SPT. Our study aimed not only to evaluate allergenic skin reactivity, but we designed this study to collaborate with the generation of some therapeutic action on allergy development since SPT can exert this role for the patient [5]. The SPT results were individually discussed in terms of social, biological, and environmental aspects. They were interviewed six months later to generate evidence about this role.

Our results demonstrated that almost $70 \%$ of the atopic volunteers could report a substantial improvement in their health after being informed about SPT results. What was highly significant compared to the control non-atopic group was that almost none reported improved health. Furthermore, near the third part of atopic individuals also reported avoiding clinical symptoms of allergic reactions while, as expected, almost all non-atopic volunteers could maintain the absence of allergic reactions. Considering that all atopic individuals were recruited with the inclusion criteria of confirmed clinical symptoms, this result strength the suggested health improvement.

Together, those observations strongly corroborate with the hypothesis of SPT exerting some therapeutic role for the patient.

We further suggest that this could result from acquiring knowledge about allergens individually capable of triggering allergy symptoms yielding the avoidance of contact with specific sources of allergens and consequently preventing allergic manifestations. This effect is described mainly for food allergies, with few observations that evaluated inhaled allergens.

Regardless of the type of allergen, the precise diagnoses can be directly related to allergen avoidance but usually requires complex allergy tests as oral food challenge [14] and the nasal allergen challenge [15] that are not easy to access in developing countries. Furthermore, SPT is the most common test for allergy diagnosis [14], suggesting that our observations had broad application in allergic disease management. 
These observations are rare in the literature where usually therapeutic aspects discuss mainly the effect of oral and epicutaneous immunotherapies, although the SPT was performed and demonstrated high sensitivity [15].

We also repeated the SPT at the therapeutic interview and obtained new blood samples to compare these parameters with the initial evaluation. Briefly, both tests and all parameters demonstrated some reduction near a $30 \%$ rate. These results corroborate with the suggestion of reduced clinical symptoms, as evidenced by the interview. As expected, the volunteers could not substantially negate the reaction observed by the SPT. Still, the literature already described that skin reactivity hardly becomes negative in a six-month follow-up [16].

Finally, it is essential to highlight that we could not find similar literature approaches where the indoor permanence time was considered an inclusion criterion to elaborate a deep discussion about our observation. Additionally, we also need to consider that the world pandemic state that started right after the conclusion of this study had lasted more than a year and that several countries have adopted social isolation to control the dissemination of COVID-19; our data may acquire great importance in the coming years.

\section{Conclusions}

Some limitations of our study need to be indicated, and those include that we did note evaluated that avoiding allergens and changing social habits could influence the quality of life in our volunteers. Although, our results suggest that SPT followed by social, biological, and environmental guidance individually performed and using WAO avoiding allergens as a reference can efficiently collaborate as a therapeutic tool preventing allergy development in individuals that remain in indoor conditions.

Supplementary Materials: The following are available online at https:/ / www.mdpi.com/article / 10.3390 /biologics1020014/s1, Figure S1. An eschamatic illustration of the therapeutic effectiveness evaluation of SPT used in our study, Table S1. Characteristics of the allergen extracts used in SPT, Table S2. Characteristics of the allergen extracts used in multiplex immunoblot assay (EUROLINE). Table S3. Comparative SPT results obtained before and six months after in-person discussion about allergen avoidance.

Author Contributions: Methodology and investigation, B.Y.N., A.P.F., D.d.S., L.S.S., T.R.d.S.; Conceptualization, writing - original draft preparation and supervision, J.R.V. All authors have read and agreed to the published version of the manuscript.

Funding: This research was funded by Sao Paulo Research Foundation (FAPESP) grant \#2018/05181-7.

Institutional Review Board Statement: The study was conducted according to the guidelines of the Declaration of Helsinki, and approved by the Institutional Ethics Committee with the following Certificate of Presentation of Ethical Appreciation: 80280417.8.0000.8114.

Informed Consent Statement: We obtained informed consent from all volunteers recruited in this study.

Acknowledgments: We thank Sérgio Luiz Teixeira Santos for technical support performing EUROLINE immunoblot assays.

Conflicts of Interest: The authors declare that they have no relevant conflicts of interest.

\section{References}

1. Popov, T.A.; Passalacqua, G.; González-Díaz, S.N.; Plavec, D.; Braido, F.; García-Abujeta, J.-L.; Dubuske, L.; Rouadi, P.; MoraisAlmeida, M.; Bonini, S.; et al. Medical devices in allergy practice. World Allergy Organ. J. 2020, 13. [CrossRef]

2. Ansotegui, I.J.; Melioli, G.; Canonica, G.W.; Caraballo, L.; Villa, E.; Ebisawa, M.; Passalacqua, G.; Savi, E.; Ebo, D.; Gómez, R.M.; et al. IgE allergy diagnostics and other relevant tests in allergy, a World Allergy Organization position paper. World Allergy Organ. J. 2020, 13, 100080. [CrossRef] [PubMed]

3. Oppenheimer, J.; Nelson, H.S. Skin testing. Ann. Allergy Asthma Immunol. 2006, 96 (Suppl. 1), S6-S12. [CrossRef]

4. Bousquet, J.; Heinzerling, L.; Bachert, C.; Papadopoulos, N.; Bousquet, P.J.; Burney, P.; Canonica, G.W.; Carlsen, K.H.; Cox, L.; Haahtela, T.; et al. Practical guide to skin prick tests in allergy to aeroallergens. Allergy 2011, 67, 18-24. [CrossRef] [PubMed] 
5. $\quad$ Bernstein, I.L.; Li, J.T.; Bernstein, D.I.; Hamilton, R.; Spector, S.L.; Tan, R.; Sicherer, S.; Golden, D.B.; Khan, D.A.; Nicklas, R.A.; et al. Allergy Diagnostic Testing: An Updated Practice Parameter. Ann. Allergy Asthma Immunol. 2008, 100 (Suppl. 3), S1-S148. [CrossRef]

6. Chen, K.-W.; Zieglmayer, P.; Zieglmayer, R.; Lemell, P.; Horak, F.; Bunu, C.P.; Valenta, R.; Vrtala, S. Selection of house dust mite-allergic patients by molecular diagnosis may enhance success of specific immunotherapy. J. Allergy Clin. Immunol. 2019, 143, 1248-1252.e12. [CrossRef] [PubMed]

7. Heinzerling, L.; Mari, A.; Bergmann, K.; Bresciani, M.; Burbach, G.; Darsow, U.; Durham, S.; Fokkens, W.; Gjomarkaj, M.; Haahtela, T.; et al. The skin prick test-European standards. Clin. Transl. Allergy 2013, 3, 3. [CrossRef] [PubMed]

8. Pastorino, A.; Kuschnir, F.; Arruda, L.; Casagrande, R.; de Souza, R.; Dias, G.; Silveira, H.; da Cunha, A.; Jacob, C.; Solé, D. Sensitisation to aeroallergens in Brazilian adolescents living at the periphery of large subtropical urban centres. Allergol. Immunopathol. 2008, 36, 9-16. [CrossRef] [PubMed]

9. Bousquet, P.J.; Chinn, S.; Janson, C.; Kogevinas, M.; Burney, P.; Jarvis, D. Geographical variation in the prevalence of positive skin tests to environmental aeroallergens in the European Community Respiratory Health Survey I. Allergy 2007, 62, 301-309. [CrossRef] [PubMed]

10. Oncham, S.; Udomsubpayakul, U.; Laisuan, W. Skin prick test reactivity to aeroallergens in adult allergy clinic in Thailand: A 12-year retrospective study. Asia Pac. Allergy 2018, 8, e17. [CrossRef] [PubMed]

11. Alcantara-Neves, N.M.; Veiga, R.V.; Ponte, J.C.M.; Da Cunha, S.S.; Simões, S.M.; Cruz, A.A.; Yazdanbakhsh, M.; Matos, S.M.; Silva, T.M.; Figueiredo, C.A.; et al. Dissociation between skin test reactivity and anti-aeroallergen IgE: Determinants among urban Brazilian children. PLoS ONE 2017, 12, e0174089. [CrossRef]

12. Khazaei, H.A.; Khazaei, B.; Dashtizadeh, G.A.; Mohammadi, M. Cigarette Smoking and Skin Prick Test in Patients With Allergic Rhinitis. Int. J. High Risk Behav. Addict. 2015, 4, e23483. [CrossRef]

13. Saleem, N.; Waqar, S.; Shafi, A. Skin Prick Test Reactivity to Common Aeroallergens among Allergic Rhinitis Patients. J. Coll. Physicians Surg. Pak. 2018, 28, 766-771. [PubMed]

14. Frati, F.; Incorvaia, C.; Cavaliere, C.; Di Cara, G.; Marcucci, F.; Esposito, S.; Masieri, S. The skin prick test. J. Biol. Regul. Homeost. Agents 2018, 32 (Suppl. 1), 19-24. [PubMed]

15. Foong, R.-X.; Dantzer, J.A.; Wood, R.A.; Santos, A.F. Improving Diagnostic Accuracy in Food Allergy. J. Allergy Clin. Immunol. Pract. 2021, 9, 71-80. [CrossRef] [PubMed]

16. Pesonen, M.; Kallio, M.J.; Siimes, M.A.; Ranki, A. Allergen Skin Prick Testing in Early Childhood: Reproducibility and Prediction of Allergic Symptoms into Early Adulthood. J. Pediatr. 2015, 166, 401-406.e1. [CrossRef] [PubMed] 